\title{
Nécessité pour la recherche de soutenir l'émergence de systèmes de santé animale non conventionnels
}

\author{
J. Domenech (1), P. Bonnet ${ }^{(2)} \&$ J.-F. Renard ${ }^{(2)}$ \\ (1) Organisation des Nations unies pour I'alimentation et l'agriculture, Viale delle Terme di Caracalla, 00100 \\ Rome, Italie \\ (2) Centre de coopération internationale en recherche agronomique pour le développement, TA 30/B, Campus \\ international de Baillarguet, 34398 Montpellier Cedex 5, France
}

\section{Résumé}

Les maladies animales restent l'un des principaux problèmes posés aux productions animales, tant pour le développement du commerce que pour la lutte contre la pauvreté et la santé publique. Les systèmes de santé animale sont complexes par la diversité des acteurs et par l'évolution du contexte, marqué notamment par la redéfinition des rôles des secteurs privé et public. II est donc souvent difficile d'évaluer les performances globales des systèmes et soussystèmes de santé animale, tant pour leur efficacité médicale qu'économique et sociale. De plus, la nécessaire fiabilité des informations sanitaires obtenues amène à la certification des statuts sanitaires des régions et des pays, ce qui impose un fort degré de normalisation et de conformité aux normes internationales. Un bilan des systèmes non conventionnels doit donc être fait et comparé à celui des systèmes conventionnels.

Un système de santé animale se conçoit dans son ensemble et ses performances doivent être évaluées de façon globale, en fonction des marchés des produits et en tenant compte des objectifs et intérêts parfois contradictoires des acteurs.

Les besoins et axes de recherches sont donc nombreux, pouvant notamment porter sur :

- les méthodes, données et outils nécessaires à l'évaluation de l'efficacité de systèmes, avec une définition d'indicateurs fiables,

- la démonstration qu'il existe un déterminisme de l'état de santé à venir des troupeaux, permettant ensuite de dresser des cartes de marqueurs de risque et de besoins en services zoosanitaires,

- la conception et gestion de systèmes d'information sanitaire réalistes et harmonisés fournissant des mesures fiables des indicateurs de santé,

- la fonction, l'organisation et l'efficacité des approches participatives de surveillance,

- la définition et l'efficacité des contrats en santé animale, tels les mandats sanitaires entre État et vétérinaires privés,

- la fonction et le rôle des auxiliaires d'élevage,

- l'élaboration de méthodes d'évaluation et de normes tenant compte des spécificités des pays du Sud et pouvant mener à la certification et à l'accréditation des systèmes non conventionnels.

II y aura ensuite lieu de tester, à l'aide des indicateurs définis précédemment, l'efficience des systèmes (impacts directs, études coûts-bénéfices) puis en tirer une " boîte d'outils " capable de guider leur mise en place, en considérant leurs avantages et inconvénients en fonction des contextes locaux.

La recherche sur l'évaluation des systèmes de santé animale constitue un investissement à long terme, mais elle est le gage d'une certification de qualité, permettant le développement et la sécurité des marchés des produits animaux.

\section{Mots-clés}

Auxiliaire d'élevage - Indicateur zoosanitaire - Mandat sanitaire - Recherche - Système non conventionnel - Système de santé animale. 


\section{Introduction}

Puissant facteur de diversification des agricultures familiales, source de revenus, outil de capitalisation et de crédit, lélevage occupe, par ses fonctions multiples et son rôle social et culturel, une place pivot dans la vie des sociétés rurales dans les pays du Sud.

Or, dans le cadre général d'augmentation des productions, de lutte contre la pauvreté et de développement du commerce des animaux et produits d'origine animale à toutes les échelles, les maladies animales constituent un véritable goulet d'étranglement et posent donc un grave problème. Par ailleurs, les crises sanitaires alimentaires chez l'homme sont en majorité liées à des agents microbiens pathogènes pour les animaux. Il y a donc une dimension de santé publique forte dans la problématique de la santé animale.

Les systèmes de santé animale sont donc forcément complexes, du fait de la diversité des acteurs impliqués. De plus, dans un contexte évolutif, marqué en particulier par les politiques d'ajustement structurel et la redéfinition des rôles des secteurs privé et public, on assiste depuis une quinzaine d'années dans les pays du Sud à la mise en place de schémas nombreux et variés, aboutissant à la coexistence de sous-systèmes de santé animale, en interaction au sein d'un même territoire national et à fortiori international $(1,5,11,13,25)$. Les résultats sont souvent difficiles à interpréter, parfois contradictoires selon le point de vue que l'on privilégie ; les performances globales sont évaluées de manières assez diverses et peu normatives, tant l'interaction entre sous-systèmes peut être variée et complexe.

En matière de santé animale et de santé publique, l'efficacité médicale, économique et sociale de la lutte contre les maladies ainsi que la fiabilité de la certification des statuts sanitaires des régions et des pays imposent un fort degré de normalisation et une conformité aux normes internationales édictées par les organismes de référence (l'OIE [Organisation mondiale de la santé animale] et l'Organisation des Nations unies pour l'alimentation et l'agriculture [FAO] en premier lieu). Il est en outre impératif que les évolutions ne marginalisent pas encore plus les petits éleveurs et les agriculteurs-éleveurs les plus pauvres ou exclus des systèmes actuels $(8,27,29)$. Ainsi un effort particulier doit être fait pour que les nouveaux systèmes garantissent à ces éleveurs l'accessibilité aux soins vétérinaires et, finalement, leur accès aux marchés certifiés de produits animaux ; inversement, l'accessibilité des soins aux éleveurs offre aux pouvoirs publics la garantie d'accéder aux informations épidémiologiques (9).

Il faut donc qu'un bilan soit fait et que les systèmes non conventionnels soient étudiés de façon beaucoup plus approfondie, parallèlement à une évaluation des systèmes conventionnels. Il y a là un champ sur lequel la recherche sur les systèmes de santé animale doit s'investir de façon beaucoup plus volontariste, en suivant l'exemple tracé en santé humaine $(7,14,15,16)$.

\section{Évolution des systèmes de santé animale}

Les grandes fonctions impliquées dans l'offre de prestation des professionnels publics et privés de santé ont été redéfinies, de même que les différentes catégories d'acteurs pouvant intervenir, depuis les docteurs vétérinaires jusqu'aux éleveurs et leurs groupements, en passant par les techniciens ou infirmiers d'élevage et les paravétérinaires ou auxiliaires délevage (4, 10, 17, 26).

Les positionnements respectifs de ces acteurs professionnels et les modalités de financements par l'État et par les éleveurs des fonctions sanitaires au sein du système de santé ont été également analysés (23).

Plusieurs études et enquêtes ont tenté d'analyser les premiers résultats de ces évolutions et d'apprécier leurs répercussions sur l'offre et la demande de services vétérinaires, ainsi que leurs conséquences sur le secteur de l'élevage et sur la commercialisation des produits d'origine animale. Les conclusions ne sont pour l'instant pas suffisamment démonstratives et limpact sur les productions animales reste peu documenté du fait de l'absence de suivis zootechniques de terrain. L'impact sur les revenus des éleveurs reste mal connu ou n'a fait l'objet que d'évaluations ponctuelles.

Pourquoi est-il aussi difficile de démontrer de façon univoque l'efficacité d'un système de santé animale donné ? Pourquoi tant de bilans mitigés, ou souvent apparemment négatifs? Le retour au « tout-État » étant exclu en raison des difficultés de financement pérenne, comment lever ou gérer les obstacles qui gênent une mise en place efficace de systèmes basés sur les lois du marché ou mixtes?

On sait que, dans le cas particulier de la santé animale et de la santé humaine, les «imperfections » du marché sont liées notamment à l'intervention de l'État (aux plans national, local, inter-régional et international), à l'existence d'externalités (positives ou négatives), à l'asymétrie de l'information et au rôle important de l'incertitude pesant sur les différents risques associés (risque sanitaire, risque d'apparition des maladies, risque économique, incertitude du bénéfice résultant de l'utilisation du service) $(18,24)$. Les auteurs de la nouvelle économie institutionnelle ont particulièrement étudié ces aspects de la régulation économique.

Un système de santé est un objet multi-acteurs qui doit répondre aux objectifs dictés par tous ses acteurs, et en conséquence la qualité d'un système est par essence complexe 
et multidimensionnelle (6). Le fonctionnement du système peut se représenter comme une chaîne d'interactions entre les acteurs qui sont les fournisseurs de soins, les usagers, et les institutions qui régulent et participent souvent au financement du système. Un système de santé doit se concevoir dans son ensemble; sa performance globale doit être envisagée en fonction des marchés et filières des produits animaux, dans un contexte de portée internationale, nationale ou locale, et en tenant compte des objectifs et intérêts parfois contradictoires tels que la lutte contre la pauvreté (équité, accessibilité et recours aux soins), la sécurité sanitaire des aliments, la compétitivité des filières agroalimentaires dans les marchés régulés par des accords et arbitrages (barrières sanitaires, etc.). Ces objectifs sont en fait le plus souvent complémentaires.

La délivrance de services aux éleveurs pose des problèmes qui se réfèrent d'abord à la forme institutionnelle idéale. On entend ici par forme institutionnelle l'institution économique la plus apte à optimiser et coordonner l'échange économique ciblé : marché libre (secteur privé : entreprises, coopératives), hiérarchie (État, institutions para-étatiques), formes mixtes de coordination (conventions, contrats). Ces problèmes concernent également le type d'organisation adéquat (agencement des acteurs, relation de réseau), le type de gestion satisfaisant (niveau de profit, type de financement avec recouvrement des coûts, subventions) et les formes relationnelles entre les acteurs (fréquence et types de contacts, barrières culturelles) (28). L'utilisation d'auxiliaires d'élevage en particulier fait l'objet depuis quelques années d'un intérêt renaissant, particulièrement dans l'objectif de combler les lacunes laissées par la transition institutionnelle des services après les plans d'ajustement structurel. Le panorama global que l'on pourrait faire sur de tels schémas et sur l'évaluation de leur impact est complexe car la responsabilité de ces auxiliaires et leur place sont encore à définir, et leur statut le plus souvent temporaire.

Il convient également d'approfondir la place des contrats dans les systèmes de santé, pour les auxiliaires d'élevage, mais aussi pour d'autres acteurs du système de santé détenteurs du mandat sanitaire. Ce dernier concept est en effet de plus en plus utilisé pour l'attribution, sous contrôle des services publics officiels, de tâches ou d'objectifs chiffrés relevant des responsabilités de l'État (vaccination contre les maladies épizootiques réglementées, épidémiosurveillance, contrôle de qualité des produits animaux) (20).

\section{Besoins et axes de recherche}

Pour soutenir l'émergence de systèmes de santé animale non conventionnels, il faut notamment que l'efficacité médicale, économique, sociale et environnementale en soit démontrée. Il y a là un besoin de recherche car les méthodes, données et outils nécessaires à cette évaluation manquent ou n'ont pas été testés ou adaptés à la santé animale, alors qu'ils ont connu un fort développement en santé humaine (19).

Les axes de recherche suivant sont dès lors à envisager :

a) Un des axes de recherche majeur serait l'identification et la mise au point d'une nomenclature de critères et d'indicateurs fiables sur l'efficacité et sur le fonctionnement des systèmes de santé tant conventionnels que non conventionnels, en termes médicaux, économiques, sociaux et environnementaux (22). On décrit en général des indicateurs d'activité (fréquentation), de structure (capacité et ressources), de processus (pratiques professionnelles lors de la prise en charge d'une fonction sanitaire donnée) et de résultats (résultats intermédiaires, par exemple taux de vaccination; résultats finaux, par exemple changement d'état de santé, morbidité et mortalité, satisfaction des usagers). Ces indicateurs seraient autant de critères d'information permettant les études de caractérisation, de conformité aux recommandations et aux normes professionnelles et de qualité des services, et des comparaisons.

b) Évaluer par un indice synthétique simple quelles sont les régions où les systèmes alternatifs se justifient le plus. Cet indice devrait être basé sur les déterminants d'un état de santé animale à venir des troupeaux, plutôt que sur l'état de santé actuel, et donc sur des cartes de besoins plutôt que des cartes d'état de santé constaté. Cet indice devra privilégier les déterminants de la santé future sur lesquels une action est possible justifiant la mise en place de services non conventionnels. Ces déterminants de la santé espérée devraient selon nous aborder les dimensions de l'immunisation durable, de l'accès aux services, et de l'éducation et la promotion sanitaire.

c) La conception et la gestion de systèmes d'information sanitaire ou médico-économique (2) réalistes mais harmonisés et donc capables de fournir des mesures fiables et universelles de ces indicateurs apparaît alors comme une étape importante avant d'aborder les séquences suivantes. Il existe un certain nombre de réseaux de surveillance sanitaire dans les pays du Sud. Un recensement et une analyse critique de ces systèmes d'information est possible, afin d'identifier ceux qui paraissent les plus appropriés aux différentes régions, systèmes d'élevage, niveaux de performances (en termes de productivité et d'efficacité économique) ou maladies ciblées. Les approches participatives de surveillance épidémiologique ou d'évaluation de l'importance des maladies, qui font l'objet aujourd'hui de nombreux travaux, seront évaluées attentivement, compte tenu des résultats prometteurs qui semblent être obtenus, notamment dans les systèmes pastoraux d'Afrique de l'Est. Une autre étape de la recherche sera donc de participer à la mise en place de méthodes de suivi-évaluation, de tester leur efficacité propre et leur faisabilité d'une part (recherche sur les outils et méthodes), et enfin de fournir des mesures fiables de ces indicateurs et de leur variabilité pour caractériser puis évaluer l'impact réel des systèmes de santé d'autre part. 
d) Il faut également effectuer des recherches sur les contrats en santé animale, parmi lesquels on peut citer les exemples des mandats sanitaires entre l'État et les vétérinaires privés, ou les contrats liant les auxiliaires d'élevage ou autres paravétérinaires avec les groupements d'éleveurs, les éleveurs, les vétérinaires et l'État, ainsi que sur les différents types de régulation entre acteurs.

e) Il sera indispensable de procéder à une caractérisation et à des comparaisons entre systèmes, en ayant recours aux méthodes classiques (statistique, économie, géographie médicale, analyse des organisations). Ces analyses des systèmes en place et de leur évolution dans les pays du Sud (étape descriptive) aboutirait à une meilleure connaissance des agents qui y participent et des flux établis entre eux (transferts d'information, financiers, de personnes, de cheptels, de technologies médicales). Il s'agit d'une étape préalable avant de mettre en relation la performance mesurée et les caractéristiques des systèmes de santé.

f) À l'aide des indicateurs et des systèmes d'information et de suivi-évaluation définis précédemment, il y aura ensuite lieu de tester l'efficacité et l'efficience des systèmes de santé (impacts directs et études coûts-bénéfices), par exemple en termes d'impact des services mis en place sur l'amélioration de l'accessibilité financière (7) mais aussi socio-culturelle et spatiale (12) aux soins ou sur l'amélioration de la production et de la mise sur le marché, ou encore sur les répercussions économiques et financières au niveau des utilisateurs finaux que sont les éleveurs. Par ailleurs, en l'absence de résultats finaux d'efficacité technique (morbidité et mortalité), l'utilisation des indicateurs intermédiaires d'évaluation et de caractérisation des systèmes de santé peut permettre de générer des hypothèses quant à l'efficacité sanitaire et le niveau présent ou attendu de maladie.

g) Puis, dans un objectif explicatif, il faut aussi démontrer qu'au-delà d'une variabilité attendue, il existe un déterminisme particulier associant différentes mesures d'efficacité et d'efficience (par exemple l'état de santé des animaux ou l'état de richesse des régions ou de bien-être des éleveurs) avec des caractéristiques des systèmes de santé décrits par ces indicateurs. h) De ces études et des comparaisons sur les systèmes ayant fait leurs preuves dans d'autres contextes, dans les pays du Nord notamment, on pourrait tirer une « boîte d'outils » capable de guider la mise en place des systèmes non conventionnels, en considérant leurs avantages et inconvénients en fonction de certains contextes géographiques et institutionnels.

i) La question de la conformité et de l'accréditation des systèmes non conventionnels par rapport aux normes internationales éditées par l'OIE notamment est, par ailleurs, cruciale. La recherche peut contribuer à l'élaboration de méthodes d'évaluation et de normes scientifiques qui puissent tenir compte des conditions spécifiques des pays du Sud en complément des normes génériques élaborées en vertu de fonctions et d'objectifs affichés pour les systèmes de soins. Il s'agirait globalement d'une nouvelle approche de l'économie de la santé vétérinaire. En effet, l'évaluation de ces systèmes peut être normative et répondre à un consensus professionnel ou idéologique sans bases scientifiques prouvées, ou être seulement basée sur la démonstration scientifique de son efficacité, sans être pour autant validée ni socialement adoptée par les acteurs. Nous considérons quant à nous que les deux démarches doivent coexister.

La recherche proposée sur l'évaluation des systèmes de santé constituera toujours un investissement sur le long terme. Elle représentera finalement un enjeu majeur relatif à la qualité du service médical rendu, à la bonne diffusion des technologies médicales et à l'organisation optimale du système de soins. Elle sera le gage d'une certification de qualité ouvrant les portes des marchés des produits animaux. Elle pourrait nécessiter l'adoption d'un consensus sur une nouvelle stratégie globale de la recherche en santé animale (comme cela a été entrepris en santé humaine) (3), qui tienne compte de ces exigences nouvelles et des réflexions récentes faites en ce sens (21). 


\title{
The need for research to support the emergence of alternative animal health systems
}

\author{
J. Domenech, P. Bonnet \& J.-F. Renard
}

\section{Summary}

Animal diseases remain one of the main problems for livestock production in terms of trade development, poverty reduction and public health. Animal health systems are complex because of the diversity of the parties involved and because of various changes in the delivery of veterinary services, such as the redefinition of the roles of the public and private sectors. It is, therefore, often difficult to assess the global performance of animal health systems and sub-systems in terms of their medical, economic and social effectiveness. In addition, the necessary reliability of the health information obtained leads to certification of the status of regions and countries with regard to epizootics, which requires a high degree of standardisation and conformity with international norms. An assessment therefore needs to be made of the advantages of alternative systems compared with conventional systems.

An animal health system should be seen as a whole, and when assessing its overall performance several things must be taken into account, e.g. the markets for products and the sometimes contradictory interests of all the different parties involved.

There are, therefore, many research needs and avenues to be pursued, including: - the methods, data and tools required for assessing the effectiveness of systems, including a definition of what constitutes a reliable indicator

- the factors that determine the health of a herd; having a clearer idea of what will affect herd health will make it possible to map risk indicators and animal health care needs

- the design and management of realistic and harmonised animal health information systems whose indicators provide reliable measurements of health

- the function, organisation and effectiveness of participative surveillance approaches

- the definition and effectiveness of animal health contracts, such as health mandates between the State and private veterinarians

- the function and role of livestock auxiliaries

- the establishment of assessment methods and standards that take into account the specific situation of southern countries that could lead to the certification and accreditation of alternative systems.

The efficiency of these systems must then be tested (direct impact, cost-benefit studies) using the above-mentioned indicators, and an implementation 'toolkit' can then be assembled, taking into account the local differences which will affect the suitability of each system for different locations.

Research into the assessment of animal health systems is a long-term investment, but it ensures that quality certification is reliable and allows for the safe development of animal product markets.

\section{Keywords}

Alternative system - Animal health accreditation mandate - Animal health indicator Animal health system - Livestock auxiliary - Research. 


\title{
Necesidad de que la investigación apoye la aparición de sistemas de sanidad animal alternativos
}

\author{
J. Domenech, P. Bonnet \& J.-F. Renard
}

\section{Resumen}

Las enfermedades animales siguen constituyendo uno de los grandes problemas de la producción animal con vistas tanto al desarrollo del comercio como a la lucha contra la pobreza y en pro de la salud pública. Los sistemas de sanidad animal son complejos por la diversidad de las partes que intervienen en ellos y por la evolución del contexto, marcado sobre todo por la redefinición de los papeles respectivos del sector público y el privado. Por ello a menudo resulta difícil evaluar el rendimiento global, en términos de eficacia médica, económica y social, de los sistemas y subsistemas zoosanitarios. Además, la necesaria fiabilidad de la información sanitaria obtenida lleva a conceder a regiones y países un determinado estatuto con respecto a ciertas epizootias, hecho que impone un alto grado de normalización y conformidad con las normas internacionales. De ahí que sea necesario estudiar a fondo los sistemas alternativos, comparando sus resultados con los de los sistemas convencionales. Un sistema de sanidad animal se concibe como un todo y su rendimiento debe evaluarse de manera global, en función de los mercados de productos y teniendo en cuenta los objetivos e intereses, a veces contradictorios, de todas las partes implicadas.

Existen pues numerosas necesidades y líneas de investigación, de entre las cuales cabe destacar las siguientes:

- métodos, datos e instrumentos necesarios para evaluar la eficacia de los sistemas, con definición de indicadores fiables;

- demostración de que existe determinismo en el estado sanitario futuro de los rebaños, lo que a su vez permite cartografiar los marcadores de riesgo y las necesidades zoosanitarias;

- concepción y gestión de sistemas de información sanitaria realizados y armonizados con aportación de medidas fidedignas de los indicadores de salud; - función, organización y eficacia de los sistemas participativos de vigilancia;

- definición y eficacia de los contratos de sanidad animal, por ejemplo las acreditaciones zoosanitarias entre Estado y veterinarios privados;

- funciones y cometido de los auxiliares zootécnicos;

- elaboración de métodos de evaluación y de normas que tengan en cuenta las características propias de los países del Sur y puedan servir para certificar y acreditar los sistemas alternativos.

Más tarde, con ayuda de indicadores predefinidos, será posible poner a prueba la eficiencia de los sistemas (repercusiones directas, estudio de la relación costo/beneficio) y concebir a partir de ahí un conjunto de instrumentos que sirva para guiar su aplicación, teniendo en cuenta sus ventajas e inconvenientes según el contexto local.

Aunque supone una inversión a largo plazo, la investigación sobre la evaluación de sistemas zoosanitarios es la vía segura hacia una certificación de calidad que haga posible el desarrollo y la seguridad de los mercados de productos de origen animal.

\section{Palabras clave}

Acreditación zoosanitaria - Auxiliar zootécnico - Indicador zoosanitario - Investigación Sistema alternativo - Sistema zoosanitario. 


\section{Bibliographie}

1. Ahuja V. (2001). - Redefining the role of government in livestock health and breeding services and managing the transition from the Status Quo to recommended State. In FAO Electronic Conference on Delivery of livestock health and breeding services in India (VetIndia). Organisation des Nations unies pour l'alimentation et l'agriculture, Rome (fao.org/Mailnews/VetIndia/htm, consulté le 15 mars 2004).

2. Bailly A. \& Périat M. (1995). - Médicométrie, une nouvelle approche de la santé. Economica, Paris, 100 pp.

3. Banque mondiale (1994). - Better health in Africa. Experience and lessons learned. Banque mondiale, Washington, DC, 260 pp.

4. Blanc P., Cori G., Kamil H., Le Masson A., Renard J.-F. \& Thonnat J. (2003). - Étude bibliographique sur les auxiliaires de santé animale. Centre de coopération internationale en recherche agronomique pour le développement Département d'élevage et de médecine vétérinaire/ Vétérinaires sans frontières, Montpellier, $73 \mathrm{pp}$.

5. Bonnet P. (2002). - Case study on Veterinary Services in Ethiopia and Kenya. In Impact of privatization of the public sector on developing countries: benefits and problems (J. Marzin, T. Voituriez \& B. Losch, édit.). Centre de coopération internationale en recherche agronomique pour le développement, Montpellier, 80-84.

6. Bonnet P. \& Duteurtre G. (2000). - An assessment of the Ethiopian animal health care system given the new tools, models and theories for economic analysis (new institutional economics, conventions theory, agency theory, contracts). In International Society for Veterinary Epidemiology and Economics (ISVEE) International Symposium, août, Breckenridge, Colorado. ISVEE, Fort Collins, Colorado, 1 p.

7. Carrin G., Perrot J. \& Sergent F. (1994). - The influence of financial participation by the population on the demand for health care. Division of intensified cooperation with countries in greatest need. Organisation mondiale de la santé, Genève, $99 \mathrm{pp}$.

8. Castro-Leal F, Dayton J., Demery L. \& Mehra K. (2000). Public spending on health care in Africa: do the poor benefit? Bull. WHO, 78 (1), 66

9. Catley A. \& Mariner J. (édit.) (2001). - Participatory epidemiology, lessons learned and future directions, 15-17 novembre, Addis Ababa. Community-based Animal Health and Participatory Epidemiology Unit (CAPE)-Programme pan-africain de contrôle des épizooties (PACE), Nairobi, 43 pp. (eldis.org/fulltext/cape_new/Addis_Ababa_PE_ Proceedings.pdf, consulté le 15 mars 2004).

10. Catley A. \& Leyland T. (édit.) (2002). - Community-based animal healthcare. PLA Notes 45. International Institute for Environment and Development (IIED), Londres, $80 \mathrm{pp}$.

11. De Haan C. \& Bekure S. (1991). - Animal health services in sub-Saharan Africa. Initial experiences with alternative approaches. World Bank Technical Paper no. 134. Banque mondiale, Washington, DC, 64 pp.
12. Deichmann U. (1997). - Accessibility indicators in GIS. Department for Economic and Social Information and Policy Analysis, United Nations Statistics Division, New York, $13 \mathrm{pp}$.

13. Deutsche Gesellschaft für Technische Zusammenarbeit (GTZ) \& Institut d'élevage et de médecine vétérinaire des pays tropicaux (IEMVT) (1988). - Vers une nouvelle structure de la santé animale en Afrique. In Politique nouvelle pour le développement de l'élevage en Afrique au sud du Sahara, 2227 février, Bangui, République Centrafricaine. IEMVT, Montpellier, $30 \mathrm{pp}$.

14. Gwatkin D.R. (2000). - Health inequalities and the health of the poor: what do we know? What can we do? Bull. WHO, 78 (1), 3

15. Johnson K.E., Kisubi W.K., Karanja Mbugua J., Lackey D., Stanfield P. \& Ben Osuga (1989). - Community based health care in Kibwezi, Kenya: 10 years in retrospect. Social Sci. Med., 28 (100), 1039-1051.

16. Kizito N. (1998). - Comprendre le secteur informel de la santé (SIS). Concepts, techniques de collecte des données et structure pour l'analyse. Organisation mondiale de la santé, Genève, 47 pp.

17. Kleeman G. (1999). - Service management in livestock development. Concepts and elements. Deutsche Gesellschaft für Technische Zusammenarbeit, Wiesbaden, $430 \mathrm{pp}$

18. Leonard D.K. (1993). - Structural reform of the veterinary profession in Africa and the new institutional economics. Dev. Change, 24, 227-267.

19. Matillon Y. \& Durieux P. (1994). - L'évaluation médicale, du concept à la pratique. Flammarion, Paris, 161 pp.

20. Perrot J., Carrin G. \& Sergent F. (1997). - L'approche contractuelle: de nouveaux partenariats pour la santé dans les pays en développement. Organisation mondiale de la santé, Genève, 69 pp.

21. Perry B.D., Randolph T.F., McDermott J.J., Sones K.R. \& Thornston P.K. (2002). - Investing in animal health research to alleviate poverty. International Livestock Research Institute, Nairobi (ilri.cgiar/InfoServ/webpub/fulldocs/ investinginanimal/Bookl/media/index.htm, consulté le 15 mars 2004)

22. Petit J. (2002). - Construction et utilisation des indicateurs dans le domaine de la santé. Principes généraux. Agence nationale d'accréditation et d'évaluation en santé, Paris, $39 \mathrm{pp}$.

23. Spore (1999). - Order in the market please! Spore, 83, 1-2.

24. Stiglitz J.E. (2000). - Information imparfaite sur le marché des biens. In Principes d'économie moderne. De Boeck Université, Bruxelles, 988 pp.

25. Tambi N.E., Mukhebi A.W., Maina O.W. \& Solomon H.M. (1997). - Privatisation of animal health services in Kenya: an evaluation of the Kenya Veterinary Association Privatisation Scheme (KVAPS). Organisation of African Unity/Inter-African 
Bureau for Animal Resources/Pan-African Rinderpest Campaign/International Livestock Research Institute (ILRI) Report. ILRI, Nairobi, 84 pp.

26. The In Development Limited (IDL) Group (2003). Community animal health workers: threat or opportunity? The IDL Group, Crewkerne, $191 \mathrm{pp}$.

27. Wiese M. \& Wyss K. (1998). - Les populations nomades et la santé humaine et animale en Afrique et notamment au Tchad. APT Reports 9. Institute of Physical Geography, Institut Tropical Suisse, Fribourg, Bâle, 117 pp.
28. Williamson O.E. (1994). - Les institutions de l'économie. Interéditions, Paris, 505 pp.

29. Zimmermann W., Pfeiffer D.U. \& Zessin K.H. (1997). Primary animal health activities in Southern Africa. Proc. International seminar, 26 février-8 mars 1996, Mzuzu, Malawi. Deutsche Stiftung für internationale Entwicklung, Zentralstelle für Ernährung und Landwirtschaft, Feldafing, $376 \mathrm{pp}$. 